\title{
Biosimilars in inflammatory bowel disease: angels or demons?
}

Keywords: crohn's disease, ulcerative colitis, inflammatory bowel disease, medical therapy, infliximab, adalimumab, anti-tnfa agents, biosimilar, ct-p13

\section{Introduction}

The inflammatory bowel diseases (IBD), Crohn's disease (CD) and ulcerative colitis (UC), are common causes of gastrointestinal illness characterized by chronic, relapsing intestinal inflammation. Their peak incidence in active and fertile period of life, the increasing prevalence and the large need in terms of investigations, medications, and hospitalization, impact consistently on the health system expenditure. ${ }^{1}$

In the last two decades, the introduction of biologic agents has revolutionized the management of IBD for patients not responding to conventional treatment. ${ }^{2}$ However, although allowing to achieve more ambitious therapeutic targets and perhaps modifying the natural history of these diseases, the drug-related cost has increased consistently. ${ }^{3}$ At the moment, there are six biological agents licensed by FDA for the treatment of CD (infliximab, adalimumab, golimumab, certolizumab, vedolizumab, and natalizumab, the last two being an anti-a $4 \mathrm{~b} 7$ and anti- $\alpha 4 \beta 1$-integrin, respectively), and three for UC (infliximab, adalimumab, and vedolizumab), whereas the EMA did not approved natalizumab for IBD. ${ }^{4}$

Thus far biologics are licensed in IBD for steroid-refractory, steroid-dependent and/or immune-modulator-refractory disease and in patients intolerant to "conventional" therapies. ${ }^{5-7}$ Beside data of randomized controlled trials (RCTs), open-label extension of RCTs and large single and multi-centres experience up to five-years with anti-TNF a agents have demonstrated that about two thirds of patients are kept in remission, although a rate of about 10-20\%/year of loss of response requires dose escalation or shift to a different biologic. ${ }^{8}$

\section{Biosimilar}

Due to their outstanding impact on the therapeutic management of many diseases, "biologics" represent one of the fastest growing segments of the pharmaceutical industry. This term broadly refers to substances produced by living cells using biotechnology (i.e. recombinant DNA technology, controlled gene expression or antibody technologies). These techniques have paved the way for tailor-made and targeted medicines. The first generation of biopharmaceutical products manufactured using recombinant technologies was launched in the 1970s-80s and they have reached or are now on the way to patent expiration. Thus, an increasing number of "copies" of these drugs have been brought or are expected to enter the market. The list includes erythropoietin, gonadotropins, granulocyte colony stimulating factors (G-CSFs), human growth hormones, human insulin, interferon, and monoclonal antibodies. ${ }^{9}$ However, these products defined as biosimilars cannot be described as mere copies of the originator drug, as it is for generics. Small distinctions in production, or even in the surrounding environment, cannot be excluded and might indeed confer differences in pharmacokinetics, treatment efficacy and safety. This is the reason why, in order to be released to the public, biosimilars must show to be as close to identical to the parent biological product based
Volume 5 Issue I - 2016

\author{
Antonio Luca Annese,Vito Annese \\ Department of Emergency, Unit of Gastroenterology, Careggi \\ University Hospital, Italy \\ Correspondence: Vito Annese, Department of Emergency, \\ Unit of Gastroenterology, Careggi University Hospital, Largo \\ Brambilla 3, 50134 Florence, Italy, \\ Email annesev@aou-careggi.toscana.it
}

Received: July 25, 2016 | Published: July 27, 2016

on data compiled not only through analytical evaluation, but also through preclinical and clinical studies: the so called comparability exercise. The same process is also applied in case of changes in the production process of the originators.

\section{Regulatory aspects}

The regulatory aspects of the comparability exercise are of particular relevance for monoclonal antibodies that are high molecular weight proteins with highly complex secondary and tertiary structures and further post-translational modifications, such as glycosylation. In October 2009 EMA and the CHMP's (Committee for Medicinal Products for Human Use) released a concept paper establishing the need for a through multidisciplinary comparability exercise for the approval of biosimilar antibody. ${ }^{10}$ Regulatory requirements indeed include structural characterization, physicochemical properties, biological activity, purity and impurities. Appropriate and sensitive methods are selected to sufficiently demonstrate comparability between the reference molecule and the biosimilar compound. This includes pharmacodynamics studies like receptor-binding assays or cell-based assays as well as animal studies. Also, a special focus is made on potential immunological responses. In addition, a clinical program for a biosimilar should have the primary aim of establishing "similarity". Usually one comparative clinical trial is required to have sufficient clinical data regarding clinical comparability between the biosimilar and the reference product. Acceptable clinical comparability margins should be pre-specified and justified according to ICH E9-Note for guidance on statistical principles for clinical trials (CPMP/ICH/363/96).

With respect to the safety, because there is a limited clinical database at the approval of a biosimilar, it is also important to collect post-approval safety data. This is because, as for all drugs, differences may not become apparent in the pre-approval period, during which only limited numbers of patients receive the product over a specified time.

Once a biosimilar has reached marketing approval, EMA and FDA 
would permit the extrapolation of data from one therapeutic indication to another, allowing the use of a biosimilar in indications for which it has not been formally studied. The rationale of extrapolation is that if the biosimilar shows adequate comparability to the innovator product for one indication, it may be reasonable to extend the approval of the biosimilar to the indications of the innovator product. A potential concern with the concept of data extrapolation is that the risks and mode of action for using a biopharmaceutical may differ in various patient population (e.g. in patients with different diseases or in adults versus children).

\section{The impact of biosimilars on ibd therapy}

The infliximab biosimilar CT-P13 (developed by CELLTRION, Inc., South Korea and marketed under the trade name Remsima ${ }^{\circledR}$ or Inflectra $\AA$ ) was the first biosimilar licensed for use in IBD in Europe, receiving approval in September 2013 and in April 2016, from the EMA and FDA, respectively. In addition, it has been the first biosimilar of a monoclonal antibody to reach the market. Since the approved indications of infliximab by the EMA and FDA are rheumatoid arthritis (AR), ankylosing spondylitis (AS), adult and paediatric Crohn's disease (CD) and ulcerative colitis (UC), psoriatic arthritis and psoriasis, the infliximab biosimilar has been licensed for all these same indications in the EU, South Korea, Japan and U.S. but not in Canada. That's because of some differences of CT-P13 and infliximab observed in vitro, potential differences in the mechanism of action of infliximab in these conditions, and the lack of clinical studies in IBD at the time of evaluation.

The clinical trials program to demonstrate biosimilarity between CT-P13 and reference infliximab consisted of a phase 1 pharmacokinetic study in patients with ankylosing spondylitis (AS), and a phase 3 study evaluating efficacy in patients with rheumatoid arthritis (RA). Both were randomized, double-blind, multinational trials with follow-up data up to 30 weeks. ${ }^{11,12}$ More recently data up to 54weeks, and subsequent open-label extension have been published. ${ }^{13-15}$ All these studies have demonstrated pharmacokinetic equivalence and clinical efficacy in both AS and AR, up to 108 weeks with an open label-extension, and also in patients switched from infliximab to CT-P13. Unfortunately, still non randomized controlled trials in IBD are available, although the results of two studies which completed the recruitment are awaited (ClinicalTrials.gov identifier: NCT02148640 and NCT02096861). For these reasons concerns have been raised by national and international societies, ${ }^{16,17}$ with regard to extrapolated indications and switching from originator in IBD patients. Subsequently, a number of studies of real-life use of CT-P13 in IBD have been published or presented at international meetings, ${ }^{18}$ demonstrating efficacy and safety comparable to infliximab.

\section{Pharmacoeconomic consideration}

A budget impact model for the introduction of CT-P13 in Germany, UK, Italy, the Netherlands, and Belgium in immune-mediated disease has been developed over a 1 -year time horizon. ${ }^{19}$ By considering only direct cost, the annual cost savings resulting from the introduction on market were projected to range from $€ 2.89$ million (Belgium, $10 \%$ discount) to $€ 33.80$ million (Germany, $30 \%$ discount). If any such savings made were used to treat additional patients, from 250 (Belgium, assuming 10\% discount) to 2602 (Germany, assuming 30\% discount) new subjects could be treated. This scenario will be further modified by the forthcoming arrival of over 20 other infliximab biosimilars and adalimumab biosimilars, since the patent for the latter will expire in 2016 and 2018 in US and EU, respectively. ${ }^{20}$ In general, it is expected that the reduced price of biosimilars can lead to cost efficiencies and drive competition also against other biologics. This in turn may benefit healthcare systems and improve patient care by increasing access to biologic therapy, or introducing other indications yet unlicensed (i.e. post-surgical recurrence in CD). It is crucial, however, that differently from the situation of generics, in the case of monoclonal biosimilars the substitution with the originator should be always performed under a careful evaluation of the treating physician and each patient.

In conclusion, the pharmaceutical market in the near future will be most probably dominated by biopharmaceuticals, whether originator or biosimilar drugs. Despite the fact that it costs much more, both in terms of money and time, to develop a biosimilar rather than a generic medicine, the potential returns for biosimilars might be massive. In addition, it is also possible that biosimilars could represent in certain circumstances an improvement on the original, but for sure they will represent either a challenge and an opportunity for physicians, patients and health services.

\section{Acknowledgments}

None.

\section{Conflicts of interest}

Author declares there are no conflicts of interest.

\section{Funding}

None.

\section{References}

1. Malik TA. Inflammatory Bowel Disease: Historical Perspective, Epidemiology, and Risk Factors. Surg Clin North Am. 2015;95(6):11051122.

2. Annese V, Duricova D, Gower-Rousseau C, et al. Impact of New Treatments on Hospitalisation, Surgery, Infection, and Mortality in IBD: a Focus Paper by the Epidemiology Committee of ECCO. J Crohns Colitis. 2016;10(2):216-225.

3. Kaplan GG. The global burden of IBD: from 2015 to 2025. Nat Rev Gastroenterol Hepatol. 2015;12(12):720-727.

4. Côté-Daigneault J, Bouin M, Lahaie R, et al. Biologics in inflammatory bowel disease: what are the data? United European Gastroenterol J. 2015;3:419-428.

5. Orlando A, Armuzzi A, Papi C, et al. The Italian Society of Gastroenterology (SIGE) and the Italian Group for the study of Inflammatory Bowel Disease (IG-IBD) Clinical Practice Guidelines: The use of tumor necrosis factor-alpha antagonist therapy in inflammatory bowel disease. Dig Liver Dis. 2011;43(1):1-20.

6. Dignass A, Lindsay JO, Sturm A, et al. Second European evidencebased consensus on the diagnosis and management of ulcerative colitis part 2: current management. J Crohns Colitis. 2012;6(10):991-1030.

7. Dignass A, Van Assche G, Lindsay JO, et al. The second European evidence-based Consensus on the diagnosis and management of Crohn's disease: Current management. J Crohns Colitis. 2010;4(1):28-62.

8. D'Haens GR, Panaccione R, Higgins PD, et al. The London Position Statement of the World Congress of Gastroenterology on Biological Therapy for IBD with the European Crohn's and Colitis Organization: when to start, when to stop, which drug to choose, and how to predict response? Am J Gastroenterol. 2011;106(2):199-212.

9. Weise M, Bielsky MC, De Smet K, et al. Biosimilars: what clinicians should know. Blood. 2012;120(26):5111-5117. 
10. EMA guideline on similar biological medicinal products containing monoclonal antibodies: non-clinical and clinical issues. CHMP/ BMWP/403543/2010.

11. Park W, Hrycaj P, Jeka S, et al. A randomised, double-blind, multicentre, parallel-group, prospective study comparing the pharmacokinetics, safety, and efficacy of CT-P13 and innovator infliximab in patients with ankylosing spondylitis: the PLANETAS study. Ann Rheum Dis. 2013;72(10):1605-1612.

12. Yoo DH, Hrycaj P, Miranda P, et al. A randomised, double-blind, parallel-group study to demonstrate equivalence in efficacy and safety of CT-P13 compared with innovator infliximab when co- administered with methotrexate in patients with active rheumatoid arthritis: the PLANETRA study. Ann Rheum Dis. 2013;72(10):1613-1620.

13. Yoo DH, Racewicz A, Brzezicki J, et al. A phase III randomized study to evaluate the efficacy and safety of CT-P13 compared with reference infliximab in patients with active rheumatoid arthritis: 54-week results from the PLANETRA study. Arthritis Res Ther. 2016;18:82.

14. Park W, Yoo DH, Miranda P, et al. Efficacy and safety of switching from reference infliximab to CT-P13 compared with maintenance of CT-P13 in ankylosing spondylitis: 102-week data from the PLANETAS extension study. Ann Rheum Dis. 2016;2015-208783.
15. Yoo DH, Prodanovic N, Jaworski J, et al. Efficacy and safety of CTP13 (biosimilar infliximab) in patients with rheumatoid arthritis: comparison between switching from reference infliximab to CT-P13 and continuing CT-P13 in the PLANETRA extension study. Ann Rheum Dis. 2016;2015-208786.

16. Annese V, Vecchi M, Italian Group for the Study of IBD (IG-IBD) Use of biosimilars in inflammatory bowel disease: Statements of the Italian Group for Inflammatory Bowel Disease. Dig Liver Dis. 2014;46(11):963-968.

17. Danese S, Gomollon F, Governing Board and Operational Board of ECCO. ECCO position statement: the use of biosimilar medicines in the treatment of inflammatory bowel disease [IBD]. J Crohns Colitis. 2013;7(7):586-589.

18. Ben-Horin S, Casteele NV, Schreiber S, et al. Biosimilars in Inflammatory Bowel Disease: Facts and Fears of Extrapolation. Clin Gastroenterol Hepatol. 2016; (16):30212-30219.

19. Jha A, Upton A, Dunlop WC, et al. The Budget Impact of Biosimilar Infliximab (Remsima $\left.{ }^{\circledR}\right)$ for the Treatment of Autoimmune Diseases in Five European Countries. Adv Ther. 2015;32(8):7427-56.

20. Udpa N, Million RP. Monoclonal antibody biosimilars. Nat Rev Drug Discov. 2016;15(1):13-14. 